\section{New PhD Programme on TA in Portugal}

\section{by António Brandão Moniz, Universidade Nova de Lisboa (Portugal)}

To offer students high quality training in research the Universidade Nova de Lisboa (Portugal) recently approved its $\mathrm{PhD}$ Programme on Technology Assessment (PDAT). It is developed at the Faculty of Sciences and Technology (FCT-UNL). It is a " 3 rd cycle graduate programme" proposed in the context of the European Union "Bologna Treaty" reformulations of higher education. The collaboration with the University of Karlsruhe is prepared.

\section{Aims}

PDAT intends to prepare researchers and experts with ability to perform autonomous research activities as well as leading innovation processes fostering economic growth and development. And the programme introduces the methodologies and analysis processes of innovation and technological development impacts in a general context of its integration in the European and international frameworks.

This 3rd educational cycle complements the basic education of the candidates (1st and 2nd cycles, or BSc/BA and MA/MSc). It develops the studies and the research and development in emergent knowledge fields. The specific aims can be considered as proposes for the organization of this programme, as the need to develop competences and capacities to analyse critically, to assess and synthesize new and complex ideas in a context of fast technological and socio-organizational changes. Another important aim is to provide knowledge at an advanced level and to develop research skills on knowledge, technology and innovation management, specifically concerning innovation acquisition processes or innovation generation, as well the practical implementation of intra-organizational innovations or in the markets served by the organizations.

The organization of this $\mathrm{PhD}$ programme also intends to promote the participation in processes of integration of advanced knowledge of "Technology Assessment" in innovative projects that aims evaluation of technological and complex systems impacts, and the establishment of new knowledge-intensive companies, either in industry or in services, of innovation results or entrepreneurs initiatives, of already established firms or of academical organizations.

Finally such $\mathrm{PhD}$ programme tries to provide advanced training to allow the qualified participation in processes of formulation, implementation, execution, follow-up and assessment of Science \& Technology policies and strategies at the level of "Technology Assessment" and innovation. Not only in Portugal but elsewhere, once the programme is open to foreign students. The $\mathrm{PhD}$ Programme provides emphasis for a development of an original research work, which must lead to the generation of new knowledge, and the capacity of handling the correspondent methodologies of scientific research. PDAT aims also to give to the candidates an important group of additional competences and skill that are essential requisites for the new challenges in a $\mathrm{PhD}$ diploma holder career.

According to recommendations of European University Association, this studies cycle covers a wide group of competences designated as "soft skills"/"transferable skills" and includes communication and technical writing competences, leadership and time and resources management, promotion of partnerships and team working, scientific ethics and intellectual propriety rights, organization of technical events, entrepreneurship and research results transfer to the socio-economical fabric, and so forth.

Scientific understanding and high level handling of research methods will contribute to develop the critical capability, the synthesis ability complexity and large dynamics context, preparing the candidates for an autonomous acting and performance. After concluding the studies cycle, the candidates will be able to acquire, with scientific rigour and technological and methodological up-dates, the capacities of intervening in processes of production, follow-up and application of knowledge of Technology Assessment in organizations.

Technology Assessment is present in a wide spectrum of companies in very different sectors of industry and services. This Doctorate course is an alternative and advanced training 
scheme of large potential for technicians that are related with processes of technological choice or technological investments. This type of technicians that will accomplish such programme are expected to work already in the public administration offices (large national laboratories, or governmental entities with specialisation in these fields) as well in large sized companies where that type of option can be placed for technological re-conversion processes, or for entrepreneurial development. But the Technology Assessment $\mathrm{PhD}$ diplomed professionals can also work in companies of medium of small-sized dimensions, but that work with anticipation of technological development processes (as for example, biotechnology, nanotechnology, micro-electronics) or of technological consultancy.

\section{Prospective professional activities}

The Technology Assessment $\mathrm{PhD}$ diplomed professionals will have additional opportunities at research centres or research and development units associated to public and private institutions, or even at high education institutions. There are also important professional opportunities in other countries of the European area. More concretely, Technology Assessment at the EPTA (European Parliamentary Technology Assessment) could for example make policy analysis about:

1. the energy situation

2. working conditions in the light of increasing ICT work

3. globalisation and labour market competences

4. GMO and environment

5. privacy in e-government

6. potential of nanotechnology in health care

It is also possible to develop $\mathrm{PhD}$ thesis in such similar topics or about renewable energy systems, the "green" chemistry, robotics and autonomous systems, new materials and nanotechnologies, or even on transport systems and mobility.

\section{Programme structure}

The PhD Programme on "Technology Assessment" is organized over a 4 year structure, and the training courses take place during the first 2 years, although during that period is supposed to start the research process leading to the final thesis. The candidate will perform his/her research in the context of research projects of one of the research centres at FCT-UNL. The IET research centre will be one of the centres that will support that research.

Students who achieve distinction in their coursework are invited to prepare a $\mathrm{PhD}$ proposal, which includes the identification of a topic, discussion of methodological issues relating to the research, and where appropriate, a discussion of the data resources to be used or developed. The proposal also identifies further areas of formal (class work and workshops) and informal study to be pursued in the course of dissertation research and writing. The $\mathrm{PhD}$ proposed plan will approved in the first year, and discussed and re-evaluated after a public discussion of a more developed proposal. For further information to the structure of the programme see http://iet.fct.unl.pt/index.php?option=com content\&task=view\&id=232\&Itemid=271.

\section{Coordinator of this Programme}

Prof. Dr. António B. Moniz

Universidade Nova de Lisboa

Faculty of Sciences and Technology

Campus de Caparica, 2829-516 Caparica, Portugal

Email: abm@fct.unl.pt or iet@fct.unl.pt

\section{$《 》$}

